\title{
The Boundedness of Fractional Integral with Variable Kernel on Variable Exponent Herz-Morrey Spaces
}

\author{
Afif Abdalmonem ${ }^{1,2 *}$, Omer Abdalrhman ${ }^{1,3}$, Shuangping Tao ${ }^{1}$ \\ ${ }^{1}$ College of Mathematics and Statistics, Northwest Normal University, Lanzhou, China \\ ${ }^{2}$ Faculty of Science, University of Dalanj, Dalanj, Sudan \\ ${ }^{3}$ College of Education, Shendi University, Shendi, Sudan \\ Email: *afeefy86@gmail.com, humoora@yahoo.com, taosp@nwnu.edu.cn
}

Received 19 March 2016; accepted 24 April 2016; published 28 April 2016

Copyright (C) 2016 by authors and Scientific Research Publishing Inc.

This work is licensed under the Creative Commons Attribution International License (CC BY).

http://creativecommons.org/licenses/by/4.0/

(c) $\underset{\mathrm{EY}}{\mathrm{P}}$ Open Access

\section{Abstract}

In this paper, we study the boundedness of the fractional integral with variable kernel. Under some assumptions, we prove that such kind of operators is bounded from the variable exponent Herz-Morrey spaces to the variable exponent Herz-Morrey spaces.

\section{Keywords}

Fractional Integral, Variable Kernel, Variable Exponent, Herz-Morrey Spaces

\section{Introduction}

Let $0<\mu<n, \Omega \in L^{\infty}\left(\mathbb{R}^{n}\right) \times L^{r}\left(S^{n-1}\right)(r \geq 1)$ is homogenous of degree zero on $\mathbb{R}^{n}$, $S^{n-1}$ denotes the unit sphere in $\mathbb{R}^{n}$. If

i) For any $x, z \in \mathbb{R}^{n}$, one has $\Omega(x, \lambda z)=\Omega(x, z)$;

ii) $\|\Omega\|_{L^{\infty}\left(\mathbb{R}^{n}\right) \times L^{r}\left(s^{n-1}\right)}:=\sup _{x \in \mathbb{R}^{n}}\left(\int_{s^{n-1}}\left|\Omega\left(x, z^{\prime}\right)\right|^{r} \mathrm{~d} \sigma\left(z^{\prime}\right)\right)^{\frac{1}{r}}<\infty$

The fractional integral operator with variable kernel $T_{\Omega, \mu}$ is defined by

$$
T_{\Omega, \mu} f(x)=\int_{\mathbb{R}^{n}} \frac{\Omega(x, x-y)}{|x-y|^{n-\mu}} f(y) \mathrm{d} y,
$$

"Corresponding author.

How to cite this paper: Abdalmonem, A., Abdalrhman, O. and Tao, S.P. (2016) The Boundedness of Fractional Integral with Variable Kernel on Variable Exponent Herz-Morrey Spaces. Journal of Applied Mathematics and Physics, 4, 787-795. http://dx.doi.org/10.4236/jamp.2016.44089 
When $\mu \equiv 1$, the above integral takes the Cauchy principal value. At this time $\mu \equiv 0, T_{\Omega, \mu}$ is much more close related to the elliptic partial equations of the second order with variable coefficients. Now we need the further assumption for $\Omega(x, z)$. It satisfies

$$
\int_{s^{n-1}} \Omega\left(x, z^{\prime}\right) \mathrm{d} \sigma\left(z^{\prime}\right)=0, \forall x \in \mathbb{R}^{n} .
$$

For $r \geq 1$, we say Kernel function $\Omega(x, z)$ satisfies the $L^{r}$-Dini condition if $\Omega$ meets the conditions i), ii) and

$$
\int_{0}^{1} \frac{\omega_{r}(\delta)}{\delta} \mathrm{d} \delta<\infty
$$

where $\omega_{r}(\delta)$ denotes the integral modulus of continuity of order $r$ of $\Omega$ defined by

$$
\omega_{r}(\delta)=\sup _{x \in \mathbb{R}^{n},|\rho|<\delta}\left(\int_{s^{n-1}}\left|\Omega\left(x, \rho z^{\prime}\right)-\Omega\left(x, z^{\prime}\right)\right|^{r} \mathrm{~d} \sigma\left(z^{\prime}\right)\right)^{\frac{1}{r}}
$$

where $\rho$ is the a rotation in $\mathbb{R}^{n}$

$$
|\rho|=\sup _{z^{\prime} \in S^{n-1}}\left|\rho z^{\prime}-z^{\prime}\right|
$$

when $\Omega \equiv 1, T_{\Omega, \mu}$ is the fraction integral operator

$$
T_{\Omega, \mu} f(x)=\int_{\mathbb{R}^{n}} \frac{|f(y)|}{|x-y|^{n-\mu}} \mathrm{d} y .
$$

The corresponding fractional maximal operator with variable kernel is defined by

$$
M_{\Omega, \mu} f(x)=\sup _{r>0} \frac{1}{r^{n-\mu}} \int_{|x-y|<r}|\Omega(x, x-y)| \mathrm{d} y .
$$

We can easily find that when $\Omega \equiv 1, M_{\Omega}, \mu$ is just the fractional maximal operator

$$
M_{\Omega, \mu} f(x)=\sup _{r>0} \frac{1}{r^{n-\mu}} \int_{|x-y|<r}|f(y)| \mathrm{d} y .
$$

Especially, in the case $\mu \equiv 0$, the fractional maximal operator reduces the Hardy-Litelewood maximal operator.

Many classical results about the fractional integral operator with variable kernel have been achieved [1]-[4]. In 1971, Muckenhoupt and Wheeden [5] had proved the operator $T_{\Omega, \mu}$ was bounded from $L^{p}$ to $L^{q}$. In 1991, Kováčik and Rákosník [6] introduced variable exponent Lebesgue and Sobolev spaces as a new method for dealing with nonlinear Dirichet boundary value problem. Then, variable problem and differential equation with variable exponent are intensively developed. In last years, more and more researchers have been interested in the theory of the variable exponent function space and its applications. The class of Herz-Morrey spaces with variable exponent is initially defined by the author [7], and the boundedness of vector-valued sub-linear operator and fractional integral on Herz-Morrey spaces with variable exponent was introduced by authors [7] and [8]. We also note that Herz-Morrey spaces with variable exponent are generalization of Morrey-Herz spaces [9] and Herz spaces with variable exponent [10]. Recently, Wang Zijian and Zhu Yueping [11] proved the boundedness of multilinear fractional integral operators on Herz-Morrey spaces with variable exponent.

The main purpose of this paper is to establish the boundedness of the fractional integral with variable kernel from $M \dot{K}_{q_{1}, p_{1}(\cdot)}^{\alpha, \lambda}\left(\mathbb{R}^{n}\right)$ to $M \dot{K}_{q_{2}, p_{2}(\cdot)}^{\alpha, \lambda}\left(\mathbb{R}^{n}\right)$. Throughout this paper $|E|$ denotes the Lebesgue measure, $\chi_{E}$ means the characteristic function of a measurable set $S \subset \mathbb{R}^{n}$. $C$ always means a positive constant independent of the main parameters and may change from one occurrence to another.

\section{Definition of Function Spaces with Variable Exponent}

In this section we define Lebesgue spaces and Herz-Morrey spaces with variable exponent. 
Let $E$ be a measurable set in $\mathbb{R}^{n}$ with $|E|>0$. We first defined Lebesgue spaces with variable exponent.

Definition 2.1. Let $p(\cdot): E \rightarrow[1, \infty)$ be a measurable function. The Lebesgue space with variable exponent $L^{p(\cdot)}(E)$ is defined by

$$
L^{p(\cdot)}(E)=\left\{f \text { is measurable }: \int_{E}\left(\frac{|f(x)|}{\eta}\right)^{p(x)} \mathrm{d} x<\infty \text { for some constant } \eta>0\right\}
$$

The space $L_{L o c}^{p(\cdot)}(E)$ is defined by

$$
L_{\text {loc }}^{p(\cdot)}(E)=\left\{f \text { is measurable }: f \in L^{p(\cdot)}(K) \text { for all compact } K \subset E\right\}
$$

The Lebesgue spaces $L^{p(\cdot)}(E)$ is a Banach spaces with the norm defined by

$$
\|f\|_{L^{p(\cdot)}(E)}=\inf \left\{\eta>0: \int_{E}\left(\frac{|f(x)|}{\eta}\right)^{p(x)} \mathrm{d} x \leq 1\right\}
$$

We denote

$$
p_{-}=\operatorname{essinf}\{p(x): x \in E\}, \quad p_{+}=\operatorname{esssup}\{p(x): x \in E\} .
$$

Then $\mathcal{P}(E)$ consists of all $p(\cdot)$ satisfying $p_{-}>1$ and $p_{+}<\infty$.

Let $M$ be the Hardy-Littlewood maximal operator. We denote $\mathfrak{B}(E)$ to be the set of all function $p(\cdot) \in \mathcal{P}(E)$ satisfying the $M$ is bounded on $L^{p(\cdot)}(E)$.

Let $B_{k}=\left\{x \in \mathbb{R}^{n}:|x| \leq 2^{k}\right\}, C_{k}=B_{k} \backslash B_{k-1}, \chi_{k}=\chi_{B_{k}}, k \in \mathbb{Z}$

Definition 2.2. Let $\alpha \in \mathbb{R}, 0<q<\infty, p(\cdot) \in \mathcal{P}\left(\mathbb{R}^{n}\right)$ and $0 \leq \lambda<\infty$. The Herz- Morrey spaces with variable exponent $M \dot{K}_{q, p(\cdot)}^{\alpha, \lambda}\left(\mathbb{R}^{n}\right)$ is defined by

$$
\begin{gathered}
M \dot{K}_{q, p(\cdot)}^{\alpha, \lambda}\left(\mathbb{R}^{n}\right)=\left\{f \in L_{L o c}^{p(\cdot)}\left(\mathbb{R}^{n} \backslash\{0\}\right):\|f\|_{M \dot{K}_{q, p(\cdot)}^{\alpha, \lambda}\left(\mathbb{R}^{n}\right)}<\infty\right\} \\
\|f\|_{M \dot{K}_{q, p(\cdot)}^{\alpha, \lambda}\left(\mathbb{R}^{n}\right)}:=\sup _{L \in \mathbb{Z}} 2^{-L \lambda}\left\{\sum_{K=-\infty}^{L} 2^{k \alpha q}\left\|f \chi_{k}\right\|_{L^{p(\cdot)}}^{q}\right\}^{1 / q}
\end{gathered}
$$

Remark 2.1. (See [6]) Comparing the Homogeneous Herz-Morrey Spaces with variable exponent with the homogeneous Herz spaces with variable exponent, where $\dot{K}_{q(\cdot)}^{\alpha, p}\left(\mathbb{R}^{n}\right)$ is defined by

$$
\dot{K}_{q(\cdot)}^{\alpha, p}\left(\mathbb{R}^{n}\right)=\left\{f \in L_{\text {Loc }}^{q(\cdot)}\left(\mathbb{R}^{n} \backslash\{0\}\right):\|f\|_{\dot{K}_{q(\cdot)}^{\alpha, p}\left(\mathbb{R}^{n}\right)}=\left\{\sum_{k=-\infty}^{\infty} 2^{\alpha k p}\left\|f \chi_{k}\right\|_{L^{q(\cdot)}\left(\mathbb{R}^{n}\right)}^{p}\right\}^{1 / p}<\infty\right\}
$$

Obviously, $\quad \dot{K}_{q(\cdot)}^{\alpha, p}\left(\mathbb{R}^{n}\right)=M \dot{K}_{p, q(\cdot)}^{\alpha, 0}\left(\mathbb{R}^{n}\right)$

\section{Properties of Variable Exponent}

In this section we state some properties of variable exponent belonging to the class $\mathfrak{B}\left(\mathbb{R}^{n}\right)$ and $\Omega \in L^{\infty}\left(\mathbb{R}^{n}\right) \times L^{r}\left(S^{n-1}\right)$.

Proposition 3.1. (See [12]) If $p(\cdot) \in \mathcal{P}\left(\mathbb{R}^{n}\right)$ satisfies

$$
\begin{gathered}
|p(x)-p(y)| \leq \frac{-C}{\log (|x-y|)},|x-y| \leq 1 / 2 \\
|p(x)-p(y)| \leq \frac{C}{\log (e+|x|)},|y| \geq|x|
\end{gathered}
$$

then, we have $p(\cdot) \in \mathfrak{B}\left(\mathbb{R}^{n}\right)$. 
Proposition 3.2. (see [13]) Suppose that $p_{1}(\cdot) \in \mathfrak{B}\left(\mathbb{R}^{n}\right), \quad \Omega \in L^{\infty}\left(\mathbb{R}^{n}\right) \times L^{r}\left(S^{n-1}\right)$. Let $0<\mu \leq \frac{n}{\left(p_{1}\right)_{+}}$, and define the variable exponent $p_{2}(\cdot)$ by: $\frac{1}{p_{1}(x)}-\frac{1}{p_{2}(x)}=\frac{\mu}{n}$. Then we have that for all $f \in L^{p_{1}(\cdot)}\left(\mathbb{R}^{n}\right)$,

$$
\left\|T_{\Omega, \mu} f\right\|_{L^{p^{2}(\cdot)}\left(\mathbb{R}^{n}\right)}=\|f\|_{L^{p_{1}(\cdot)}\left(\mathbb{R}^{n}\right)}
$$

Now, we need recall some lemmas

Lemma 3.1. (See [14]) Given $p(\cdot): \mathbb{R}^{n} \rightarrow[1, \infty)$ have that for all function $f$ and $g$,

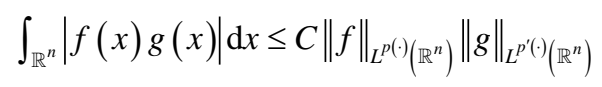

Lemma 3.2. (See [15]) Suppose that $0<\mu<n, r>1, \Omega \in L^{\infty}\left(\mathbb{R}^{n}\right) \times L^{r}\left(S^{n-1}\right)$ satisfies the $L^{r}$-Dini condition. If there exists an $0<\alpha<1 / 2$ such that $|y|<\alpha_{0} R$ then

$$
\left(\int_{R<|x|<2 R}\left|\frac{\Omega(x, x-y)}{\left.|x-y|^{n-\mu}\right)}-\frac{\Omega(x, x)}{|x|^{n-\mu}}\right|^{r} \mathrm{~d} x\right)^{\frac{1}{r}} \leq C R^{\left(\frac{n}{r}-n+\mu\right)}\left(\frac{|y|}{R}+\int_{|y| / 2 R}^{|y| / R} \frac{\omega_{r}(\delta)}{\delta} \mathrm{d} \delta\right)
$$

Lemma 3.3. (See [16]) Suppose that $x \in \mathbb{R}^{n}$, the variable function $\tilde{q}(x)$ is defined by $\frac{1}{p(x)}=\frac{1}{q}+\frac{1}{\tilde{q}(x)}$, then for all measurable function $f$ and $g$, we have

$$
\|f(x) g(x)\|_{L^{p(\cdot)}\left(\mathbb{R}^{n}\right)} \leq C\|g(x)\|_{L^{r}\left(\mathbb{R}^{n}\right)}\|f(x)\|_{L^{\hat{q}(\cdot)}\left(\mathbb{R}^{n}\right)}
$$

Lemma 3.4. (See [17]) Suppose that $p(\cdot) \in \mathfrak{B}\left(\mathbb{R}^{n}\right)$ and $0<p^{-} \leq p^{+}<\infty$.

1) For any cube and $|Q| \leq 2^{n}$, all the $\chi \in Q$, then: $\left\|\chi_{Q}\right\|_{L^{p()}} \approx|Q|^{1 / p(x)}$

2) For any cube and $|Q| \geq 1$, then $\left\|\chi_{Q}\right\|_{L^{p()}} \approx|Q|^{1 / p_{\infty}}$ where

$$
p_{\infty}=\lim _{x \rightarrow \infty} p(x)
$$

Lemma 3.5. (See [18]) If $p(\cdot) \in \mathfrak{B}\left(\mathbb{R}^{n}\right)$, then there exist constant $C>0$ such that for all balls $B$ in $\mathbb{R}^{n}$ and all measurable subset $S \subset R$

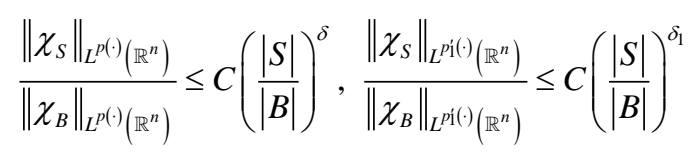

such that $\delta, \delta_{1}$ is constants satisfying $0<\delta, \delta_{1}<1$

Lemma 3.6. (See [14]) If $p(\cdot) \in \mathfrak{B}\left(\mathbb{R}^{n}\right)$, there exist a constant $C>0$ such that for any balls $B$ in $\mathbb{R}^{n}$. we have

$$
\frac{1}{|B|}\left\|\chi_{B}\right\|_{L^{p(\cdot)}\left(\mathbb{R}^{n}\right)}\left\|\chi_{B}\right\|_{L^{p^{\prime}(\cdot)}\left(\mathbb{R}^{n}\right)} \leq C
$$

\section{Main Theorem and Its Proof}

In this section we prove the boundedness of fractional integral with variable kernel on variable exponent HerzMorrey spaces under some conditions.

Theorem A. Suppose that $0<\mu<n, 0<\beta \leq 1, \lambda<\alpha<n \delta_{1}+\beta, 0<q_{1} \leq q_{2}<\infty$. Let $\Omega \in L^{\infty}\left(\mathbb{R}^{n}\right) \times L^{r}\left(S^{n-1}\right)\left(r>p_{2}^{+}\right)$, and the integral modulus of continuity $\omega_{r}(\delta)$ satisfies 


$$
\int_{0}^{1} \frac{\omega_{r}(\delta)}{\delta^{1+\beta}} \mathrm{d} \delta<\infty
$$

And let $p_{1}(\cdot) \in \mathfrak{B}\left(\mathbb{R}^{n}\right)$ satisfy $0<\mu \leq \frac{n}{\left(p_{1}\right)_{+}}$and define the variable exponent $p_{2}(x)$ by $\frac{1}{p_{1}(x)}-\frac{1}{p_{2}(x)}=\frac{\mu}{n}$, then we have

$$
\left\|T_{\Omega, \mu} f\right\|_{M \dot{K}_{q_{2}, p 2(\cdot)}^{\alpha, \lambda}\left(\mathbb{R}^{n}\right)} \leq C\|f\|_{M \dot{K}_{q_{1}, p_{1}(\cdot)}^{\alpha,}\left(\mathbb{R}^{n}\right)}
$$

For all $f \in M \dot{K}_{q_{1}, p_{1}(\cdot)}^{\alpha, \lambda}\left(\mathbb{R}^{n}\right)$.

Proof If $f \in M \dot{K}_{q_{1}, p_{1}(\cdot)}^{\alpha, \lambda}\left(\mathbb{R}^{n}\right)$ arbitrarily, we apply inequality $\left[\sum_{j=1}^{\infty} a_{j}\right]^{q_{1} / q_{2}} \leq \sum_{j=1}^{\infty} a_{j}^{q_{1} / q_{2}} \quad q_{1}, q_{2}, \cdots \geq 0$

$$
\begin{aligned}
\left\|T_{\Omega, \mu} f\right\|_{M \dot{K}_{q_{2}, p p_{2} \cdot(\cdot)}^{\alpha, \lambda}}\left(\mathbb{R}^{n}\right) & =\sup _{L \in \mathbb{Z}} 2^{-L \lambda q_{1}}\left\{\sum_{K=-\infty}^{L} 2^{k \alpha q_{2}}\left\|T_{\Omega, \mu}(f) \chi_{k}\right\|_{L^{p_{2}(\cdot)}\left(\mathbb{R}^{n}\right)}^{q_{2}}\right\}^{q_{1} / q_{2}} \\
& \leq \sup _{L \in \mathbb{Z}} 2^{-L \lambda q_{1}}\left\{\sum_{K=-\infty}^{L} 2^{k \alpha q_{1}}\left\|T_{\Omega, \mu}(f) \chi_{k}\right\|_{L^{p_{2}(\cdot)}\left(\mathbb{R}^{n}\right)}^{q_{q^{2}}}\right\}
\end{aligned}
$$

If we denote

$$
f(x)=\sum_{k=-\infty}^{\infty} f(x) \chi_{k}=\sum_{k=-\infty}^{\infty} f_{j}(x)
$$

Then we have

$$
\begin{aligned}
\left\|T_{\Omega, \mu} f\right\|_{M \dot{K}_{q_{2}, p_{2}(\cdot)}^{\alpha, \lambda}\left(\mathbb{R}^{n}\right)}^{q_{1}} \leq & \sup _{L \in \mathbb{Z}} 2^{-L \lambda q_{1}} \sum_{k=-\infty}^{L} 2^{k \alpha q_{1}}\left(\sum_{j=-\infty}^{\infty}\left\|T_{\Omega, \mu}\left(f_{j}\right) \chi_{k}\right\|_{L^{p_{2}(\cdot)}\left(\mathbb{R}^{n}\right)}\right)^{q_{1}} \\
\leq & \sup _{L \in \mathbb{Z}} 2^{-L \lambda q_{1}} \sum_{k=-\infty}^{L} 2^{k \alpha q_{1}}\left(\sum_{j=-\infty}^{k-2}\left\|T_{\Omega, \mu}\left(f_{j}\right) \chi_{k}\right\|_{L^{p_{2}(\cdot)}\left(\mathbb{R}^{n}\right)}\right)^{q_{1}} \\
& +\sup _{L \in \mathbb{Z}} 2^{-L \lambda q_{1}} \sum_{k=-\infty}^{L} 2^{k \alpha q_{1}}\left(\sum_{j=k-1}^{\infty}\left\|T_{\Omega, \mu}\left(f_{j}\right) \chi_{k}\right\|_{L^{p_{2}(\cdot)}\left(\mathbb{R}^{n}\right)}\right)^{q_{1}} \\
= & U_{1}+U_{2} .
\end{aligned}
$$

Below, we first estimate $U_{1}$ using size condition of $f_{j}$. Minkowski inequality when $j \leq k-1$, we get

$$
\begin{gathered}
\left\|T_{\Omega, \mu}\left(f_{j}\right) \chi_{k}\right\|_{L^{p_{2}(\cdot)}\left(\mathbb{R}^{n}\right)}=\left(\int_{C_{k}}\left|T_{\Omega, \mu}\left(f_{j}\right)\right|^{p_{2}(\cdot)} \mathrm{d} x\right)^{1 / p_{2}(\cdot)} \\
\left|T_{\Omega, \mu}\left(f_{j}\right)\right|=\left|\int_{\mathbb{R}^{n}} \frac{\Omega(x, x-y)}{|x-y|^{n-\mu}} f_{j}(y) \mathrm{d} y\right|
\end{gathered}
$$

Then we have

$$
\left\|T_{\Omega, \mu}\left(f_{j}\right) \chi_{k}\right\|_{L^{p_{2}(\cdot)\left(\mathbb{R}^{n}\right)}}=\int_{B_{j}} f_{j}(y)\left\|\frac{\Omega(x, x-y)}{|x-y|^{n-\mu}}-\frac{\Omega(x, x)}{|x|^{n-\mu}} \mid \chi_{k}\right\|_{L^{p^{2} \cdot(\cdot)\left(\mathbb{R}^{n}\right)}} \mathrm{d} y
$$

Since $r>p_{2}^{+}$we define the variable exponent $\frac{1}{p_{2}(x)}=\frac{1}{r}+\frac{1}{\tilde{p}_{2}(x)}$ by Lemma 3.3 and we get 


$$
\begin{aligned}
& \left\|\mid \frac{\Omega(x, x-y)}{|x-y|^{n-\mu}}-\frac{\Omega(x, x)}{|x|^{n-\mu}} \chi_{k}\right\|_{L^{p_{2}(\cdot)}\left(\mathbb{R}^{n}\right)} \\
& \leq\left\|\frac{\Omega(x, x-y)}{|x-y|^{n-\mu}}-\frac{\Omega(x, x)}{|x|^{n-\mu}}\right\|_{L^{r}\left(\mathbb{R}^{n}\right)}\left\|\chi_{k}\right\|_{L^{\tilde{p}_{2}(x)}\left(\mathbb{R}^{n}\right)} \\
& \leq\left\|\frac{\Omega(x, x-y)}{|x-y|^{n-\mu}}-\frac{\Omega(x, x)}{|x|^{n-\mu}}\right\|_{L^{r}\left(\mathbb{R}^{n}\right)}\left\|\chi_{B_{k}}\right\|_{L^{\tilde{p}_{2}(x)}\left(\mathbb{R}^{n}\right)}
\end{aligned}
$$

According to Lemma 3.4 and the formula $\frac{1}{\tilde{p}_{2}(x)}=\frac{1}{p_{2}(x)}-\frac{1}{r}$, then we have $\left\|\chi_{B_{k}}\right\|_{L_{L^{2}(x)}^{\tilde{p}\left(\mathbb{R}^{n}\right)}} \approx\left\|\chi_{B_{k}}\right\|_{L^{p_{1}(x)}\left(\mathbb{R}^{n}\right)}|B|^{\frac{-1}{r}-\frac{\mu}{n}}$. Combining Lemma 3.2, note that $2^{j-k} \leq 2^{(j-k) \beta}$, we get

$$
\begin{aligned}
\left\|\frac{\Omega(x, x-y)}{|x-y|^{n-\mu}}-\frac{\Omega(x, x)}{|x|^{n-\mu}}\right\|_{L^{r}\left(\mathbb{R}^{n}\right)} & \leq C R^{\left(\frac{n}{r}-n+\mu\right)}\left(\frac{|y|}{2^{k-1}}+\int_{|y| / 2^{k}}^{|y| / 2^{k-1}} \frac{\omega_{r}(\delta)}{\delta} \mathrm{d} \delta\right) \\
& \leq C R^{\left(\frac{n}{r}-n+\mu\right)}\left(2^{j-k}+2^{(j-k) \beta} \int_{0}^{1} \frac{\omega_{r}(\delta)}{\delta^{1+\beta}} \mathrm{d} \delta\right) \\
& \leq C R^{\left(\frac{n}{r}-n+\mu\right)} 2^{(j-k) \beta}\left(1+\int_{0}^{1} \frac{\omega_{r}(\delta)}{\delta^{1+\beta}} \mathrm{d} \delta\right) \\
& =C 2^{(k-1)\left(\frac{n}{r}-n+\mu\right)} 2^{(j-k) \beta}
\end{aligned}
$$

It follows that

$$
\left\|T_{\Omega, \mu}\left(f_{j}\right) \chi_{k}\right\|_{L^{p_{2}(\cdot)}\left(\mathbb{R}^{n}\right)} \leq C 2^{-k n+(j-k) \beta} \int_{B_{j}} f_{j}(y) \mathrm{d} y\left\|\chi_{B_{k}}\right\|_{L^{p_{1}(x)}\left(\mathbb{R}^{n}\right)}
$$

Using Lemma 3.1, Lemma 3.5 and Lemma 3.6, we obtain

$$
\begin{aligned}
& \left\|T_{\Omega, \mu}\left(f_{j}\right) \chi_{k}\right\|_{L^{p_{2}(\cdot)}\left(\mathbb{R}^{n}\right)} \leq C 2^{-k n+(j-k) \beta}\left\|f_{j}\right\|_{L^{p_{1}(x)}\left(\mathbb{R}^{n}\right)}\left\|\chi_{B_{j}}\right\|_{L^{p^{1}(\cdot)}\left(\mathbb{R}^{n}\right)}\left\|\chi_{B_{k}}\right\|_{L^{p_{1}(x)}\left(\mathbb{R}^{n}\right)} \\
& \leq C 2^{(j-k) \beta}\left\|f_{j}\right\|_{L^{p_{1}(x)}\left(\mathbb{R}^{n}\right)}\left\|\chi_{B_{j}}\right\|_{L^{P^{i}(\cdot)}\left(\mathbb{R}^{n}\right)} 2^{-k n}\left\|\chi_{B_{k}}\right\|_{L^{p_{1}(x)}\left(\mathbb{R}^{n}\right)} \\
& \leq C 2^{(j-k) \beta}\left\|f_{j}\right\|_{L^{p_{1}(\cdot)}\left(\mathbb{R}^{n}\right)} \frac{\left\|\chi_{B_{j}}\right\|_{L^{p^{1}(\cdot)}\left(\mathbb{R}^{n}\right)}}{\left\|\chi_{B_{k}}\right\|_{L^{p^{1}} \cdot(\cdot)}\left(\mathbb{R}^{n}\right)} \\
& \leq C 2^{(j-k) \beta} 2^{(j-k) n \delta_{1}}\left\|f_{j}\right\|_{L^{p_{1}(x)}\left(\mathbb{R}^{n}\right)} \\
& \leq 2^{(j-k)\left(\beta+n \delta_{1}\right)}\left\|f_{j}\right\|_{L^{p_{1}(\cdot)}\left(\mathbb{R}^{n}\right)}
\end{aligned}
$$

Hence we have

$$
\begin{aligned}
U_{1} & \leq C \sup _{L \in \mathbb{Z}} 2^{-L \lambda q_{1}} \sum_{k=-\infty}^{L}\left(\sum_{j=-\infty}^{k-2} 2^{\alpha k} 2^{(j-k)\left(\beta+n \delta_{1}\right)}\left\|f_{j}\right\|_{L^{p_{1}(x)}\left(\mathbb{R}^{n}\right)}\right)^{q_{1}} \\
& \leq C \sup _{L \in \mathbb{Z}} 2^{-L \lambda q_{1}} \sum_{k=-\infty}^{L}\left(\sum_{j=-\infty}^{k-2} 2^{\alpha j} 2^{(j-k)\left(\beta+n \delta_{1}-\alpha\right)}\left\|f_{j}\right\|_{L^{p_{1}(x)}\left(\mathbb{R}^{n}\right)}\right)^{q_{1}}
\end{aligned}
$$


Remark that $\alpha<n \delta_{1}+\beta$. We consider the two cases $1<q_{1}<\infty$ and $0<q_{1} \leq 1$. If $0<q_{1} \leq 1$, then we use the Hölder inequality and obtain

$$
\begin{aligned}
U_{1} & \leq C \sup _{L \in \mathbb{Z}} 2^{-L \lambda q_{1}} \sum_{k=-\infty}^{L}\left(\sum_{j=-\infty}^{k-2} 2^{\alpha j q_{1}} 2^{(j-k)\left(\beta+n \delta_{1}-\alpha\right)^{q_{1} / 2}}\left\|f_{j}\right\|_{L^{p_{1}(\cdot)}\left(\mathbb{R}^{n}\right)}^{q_{1}}\right) \times\left(\sum_{j=-\infty}^{k-2} 2^{(j-k)\left(\beta+n \delta_{1}-\alpha\right)^{q^{\prime}} / 2}\right)^{q_{1} / q_{1}^{\prime}} \\
& \leq C \sup _{L \in \mathbb{Z}} 2^{-L \lambda q_{1}} \sum_{k=-\infty}^{L} \sum_{j=-\infty}^{k-2} 2^{\alpha j q_{1}} 2^{(j-k)\left(\beta+n \delta_{1}-\alpha\right)^{q_{1} / 2}}\left\|f_{j}\right\|_{L^{q_{1}(\cdot)}\left(\mathbb{R}^{n}\right)}^{q_{1}} \\
& \leq C \sup _{L \in \mathbb{Z}} 2^{-L \lambda q_{1}} \sum_{j=-\infty}^{l-2} 2^{\alpha j q_{1}}\left\|f_{j}\right\|_{L^{p_{1}(\cdot)}\left(\mathbb{R}^{n}\right)}^{q_{1}} \sum_{k=j+2}^{L} 2^{(j-k)\left(\beta+n \delta_{1}-\alpha\right)^{q_{1} / 2}} \\
& \leq C \sup _{L \in \mathbb{Z}} 2^{-L \lambda q_{1}} \sum_{j=-\infty}^{l-2} 2^{\alpha j q_{1}}\left\|f_{j}\right\|_{L^{p_{1}(\cdot)}\left(\mathbb{R}^{n}\right)}^{q_{1}} \\
& \left.\leq C\|f\|_{M k_{q_{1}, p_{1}(\cdot)}(\cdot)}^{q_{1}} \mathbb{R}^{n}\right)
\end{aligned}
$$

If $0<q_{1} \leq 1, \alpha<n \delta_{1}+\beta$, we get

$$
\begin{aligned}
U_{1} & \leq C \sup _{L \in \mathbb{Z}} 2^{-L \lambda q_{1}} \sum_{k=-\infty}^{L} \sum_{j=-\infty}^{k-2} 2^{\alpha j q_{1}} 2^{(j-k)\left(\beta+n \delta_{1}-\alpha\right)^{q_{1}}}\left\|f_{j}\right\|_{L^{p_{1} \cdot(\cdot)}\left(\mathbb{R}^{n}\right)}^{q_{1}} \\
& \leq C \sup _{L \in \mathbb{Z}} 2^{-L \lambda q_{1}} \sum_{j=-\infty}^{l-2} 2^{\alpha j q_{1}}\left\|f_{j}\right\|_{L^{p^{1}(\cdot)}\left(\mathbb{R}^{n}\right)}^{q_{1}} \sum_{k=j+2}^{L} 2^{(j-k)\left(\beta+n \delta_{1}-\alpha\right)^{q_{1}}} \\
& \leq C \sup _{L \in \mathbb{Z}} 2^{-L \lambda q_{1}} \sum_{j=-\infty}^{l-2} 2^{\alpha j q_{1}}\left\|f_{j}\right\|_{L^{p_{1}(\cdot)}\left(\mathbb{R}^{n}\right)}^{q_{1}} \\
& \leq C\|f\|_{M K_{q_{1}, p p_{1}(\cdot)}^{\alpha, \lambda}\left(\mathbb{R}^{n}\right)}^{q_{q^{1}}}
\end{aligned}
$$

Next we estimate $U_{2}$, by using Proposition 3.2 we have

$$
\begin{aligned}
U_{2} \leq C \sup _{L \in \mathbb{Z}} 2^{-L \lambda q_{1}} \sum_{k=-\infty}^{L} 2^{k \alpha q_{1}}\left(\sum_{j=k-1}^{\infty}\left\|T_{\Omega, \mu}\left(f_{j}\right) \chi_{k}\right\|_{L^{p_{2}(\cdot)}\left(\mathbb{R}^{n}\right)}\right)^{q_{1}} \\
\leq C \sup _{L \in \mathbb{Z}} 2^{-L \lambda q_{1}} \sum_{k=-\infty}^{L} 2^{k \alpha q_{1}}\left(\sum_{j=k-1}^{\infty}\left\|f_{j}\right\|_{L^{p_{1}(\cdot)}\left(\mathbb{R}^{n}\right)}\right)^{q_{1}} \\
\leq C \sup _{L \in \mathbb{Z}} 2^{-L \lambda q_{1}} \sum_{k=-\infty}^{L}\left(\sum_{j=k-1}^{\infty} 2^{\alpha j} 2^{(k-j) \alpha}\left\|f_{j}\right\|_{L^{p_{1}(\cdot)}\left(\mathbb{R}^{n}\right)}\right)^{q_{1}} \\
\leq C \sup _{L \in \mathbb{Z}} 2^{-L \lambda q_{1}} \sum_{k=-\infty}^{L}\left(\sum_{j=k-1}^{k+1} 2^{\alpha j} 2^{(k-j) \alpha}\left\|f_{j}\right\|_{L^{p_{1}(\cdot)}\left(\mathbb{R}^{n}\right)}\right)^{q_{1}} \\
+C \sup _{L \in \mathbb{Z}} 2^{-L \lambda q_{1}} \sum_{k=-\infty}^{L}\left(\sum_{j=k+2}^{\infty} 2^{\alpha j} 2^{(k-j) \alpha}\left\|f_{j}\right\|_{L^{p_{1}(\cdot)}\left(\mathbb{R}^{n}\right)}\right)^{q_{1}} \\
=J_{1}+J_{2}
\end{aligned}
$$

First we estimate of $J_{1}$, then we have

$$
\begin{aligned}
J_{1} & \leq C \sup _{L \in \mathbb{Z}} 2^{-L \lambda q_{1}} \sum_{k=-\infty}^{L}\left(\sum_{j=k-1}^{k+1} 2^{\alpha j} 2^{(k-j) \alpha}\left\|f_{j}\right\|_{L^{p_{1} \cdot(\cdot)}\left(\mathbb{R}^{n}\right)}\right)^{q_{1}} \\
& \leq C \sup _{L \in \mathbb{Z}} 2^{-L \lambda q_{1}} \sum_{k=-\infty}^{L} 2^{\alpha k q_{1}}\left\|f_{k}\right\|_{L^{p_{1}}(\cdot)\left(\mathbb{R}^{n}\right)}^{q_{1}} \\
& \leq C\|f\|_{\left.M K_{q_{1}, p_{1}}^{\alpha, \cdot}()^{q_{1}}\right)}^{\left.\mathbb{R}^{n}\right)}
\end{aligned}
$$


To estimate of $J_{2}$, when $\lambda<\alpha$, we have

$$
\begin{aligned}
& J_{2} \leq C \sup _{L \in \mathbb{Z}} 2^{-L \lambda q_{1}} \sum_{k=-\infty}^{L}\left(\sum_{j=k+2}^{\infty} 2^{\alpha j} 2^{(k-j) \alpha}\left\|f_{j}\right\|_{L^{p_{1}(\cdot)}\left(\mathbb{R}^{n}\right)}\right)^{q_{1}} \\
& \leq C \sup _{L \in \mathbb{Z}} \sum_{k=-\infty}^{L} 2^{(K-L) \lambda q_{1}}\left(\sum_{j=k+2}^{\infty} 2^{(k-j)(\alpha-\lambda)} 2^{-j \lambda} 2^{j \alpha}\left\|f_{j}\right\|_{L^{p_{1}} \cdot(\cdot)\left(\mathbb{R}^{n}\right)}\right)^{q_{1}} \\
& \leq C \sup _{L \in \mathbb{Z}} \sum_{k=-\infty}^{L} 2^{(K-L) \lambda q_{1}}\left(\sum_{j=k+2}^{\infty} 2^{(k-j)(\alpha-\lambda)} 2^{-j \lambda}\left\{\sum_{n=-\infty}^{j} 2^{n \alpha}\left\|f_{n}\right\|_{L^{p_{1}}(\cdot)\left(\mathbb{R}^{n}\right)}^{q_{1}}\right\}^{\frac{1}{q_{1}}}\right)^{q_{1}} \\
& \leq C \sup _{L \in \mathbb{Z}} \sum_{k=-\infty}^{L} 2^{(K-L) \lambda q_{1}}\left(\sum_{j=k+2}^{\infty} 2^{(k-j)(\alpha-\lambda)}\right)^{q_{1}}\|f\|_{M K_{q_{1}, p_{1}(\cdot)}^{q_{1}, \cdot,}\left(\mathbb{R}^{n}\right)}^{q_{1}} \leq C\|f\|_{M \dot{K}_{q_{1}, p_{1}(\cdot)}^{\alpha, \lambda}}^{\left.q_{1}, \mathbb{R}^{n}\right)}
\end{aligned}
$$

Complete prove Theorem A.

\section{Competing Interests}

The authors declare that they have no competing interests.

\section{Acknowledgments}

This paper is supported by National Natural Foundation of China (Grant No. 11561062).

\section{References}

[1] Kenig, C. (1994) Harmonic Analysis Techniques for Second Order Elliptic Boundary Value Problems. American Mathematical Society, Providence. http://dx.doi.org/10.1090/cbms/083

[2] Calderón, A. and Zygmund, A. (1955) On a Problem of Mihilim. Transactions of the American Mathematical Society, 78, 209-224. http://dx.doi.org/10.2307/1992955

[3] Calderón, A. and Zygmund, A. (1978) On Singular Integral with Variable Kernels. Journal of Applied Analysis, 7, 221-238.

[4] Christ, M., Duoandikoetxea, J. and Rubio de Francia, J. (1986) Maximal Operators Related to the Radon Transform and the Calderóon-Zygmund Method of Rotations. Duke Mathematical Journal, 53, 189-209. http://dx.doi.org/10.1215/S0012-7094-86-05313-5

[5] Muckenhoupt, B. and Wheeden, R. (1971) Weighted Norm Inequalities for Singular and Fractional Integrals. Transactions of the American Mathematical Society, 161, 249-258. http://dx.doi.org/10.1090/S0002-9947-1971-0285938-7

[6] Izuki, M. (2010) Boundedness of Commutators on Herz Spaces with Variable Exponent. Rendiconti del Circolo Matematico di Palermo, 59, 199-213. http://dx.doi.org/10.1007/s12215-010-0015-1

[7] Izuki, M. (2010) Fractional Integrals on Herz-Morrey Spaces with Variable Exponent. Hiroshima Mathematical Journal, 40, 343-355,.

[8] Izuki, M. (2009) Boundedness of Vector-Valued Sublinear Operators on Herz-Morrey Spaces with Variable Exponent. Mathematical Sciences Research Journal, 13, 243-253.

[9] Lu, S. and Xu, L. (2005) Boundedness of Rough Singular Integral Operators on the Homogeneous Morrey-Herz Spaces. Hokkaido Mathematical Journa, 34, 299-314. http://dx.doi.org/10.14492/hokmj/1285766224

[10] Izuki, M. (2009) Herz and Amalgam Spaces with Variable Exponent, the Haar Wavelets and Greediness of the Wavelet System. East Journal on Approximations, 15, 87-109.

[11] Wang, Z. and Zhu, Y. (2014) Boundedness of Multilinear Fractional Integral Operators on Herz-Morrey Spaces with Variable Exponent. Journal of Lantong University (Natural Science Edition), 13, 60-68.

[12] Cruz-Uribe, D. and Fiorenza, A. (2013) Variable Lebesgue Spaces. Foundations and Harmonic Analysis. Springer, New York.

[13] Wu, H. and Lan, J. (2012) The Boundedness for a Class of Rough Fractional Integral Operators on Variable Exponent Lebesgue Spaces. Analysis in Theory and Applications, 28, 286-293.

[14] Tan, J. and Liu, Z. (2015) Some Boundedness of Homogeneous Fractional Integrals on Variable Exponent Function 
Spaces. ACTA Mathematics Science (Chinese Series), 58, 310-320.

[15] Cruz-Uribe, D., Fiorenza, A., Martell, J. and Pe'rez, C. (2006) The Boundedness of Classical Operators on Variable $L^{p}$ Spaces. Annales Academiae Scientiarum Fennicae Mathematica, 31, 239-264.

[16] Diening, L., Harjulehto, P., Hästö, P. and Ružičcka, M. (2011) Lebesgue and Sobolev Spaces with Variable Exponents. Springer-Verlag, Berlin Heidelberg. http://dx.doi.org/10.1007/978-3-642-18363-8

[17] Ding, Y. and Lu, S. (2000) Homogeneous Fractional Integrals on Hardy Spaces. Tohoku Mathematical Journal, 52, 153-162.

[18] Izuki, M. (2010) Boundedness of Sublinear Operators on Herz Spaces with Variable Exponent and Application to Wavelet Characterization. Analysis Mathematica, 36, 33-50. http://dx.doi.org/10.1007/s10476-010-0102-8 